\title{
Accuracy of intra-operative frozen section in the diagnosis of borderline ovarian tumors and clinical impact of underdiagnosis
}

\author{
Simone Ferrero ${ }^{1,2 *}$, Matteo Morotti ${ }^{1,2}$, Pier Luigi Venturini ${ }^{1,2}$, Leonardo Penuela ${ }^{3}$,Valerio Gaetano Vellone ${ }^{3}$ and Fabio Barra ${ }^{1,2}$ \\ ${ }^{1}$ Academic Unit of Obstetrics and Gynecology, Ospedale Policlinico San Martino, Largo R. Benzi 10, 16132 Genoa, Italy \\ ${ }^{2}$ Department of Neurosciences, Rehabilitation, Ophthalmology, Genetics, Maternal and Child Health (DiNOGMI), University of Genoa, Italy \\ ${ }^{3}$ Department of Surgical and Morphological Sciences, Division of Pathology, University of Genoa
}

\begin{abstract}
Introduction: The use of frozen section analysis is of considerable importance for the diagnosis of borderline ovarian tumors (BOTs) and to plan the most appropriate surgical management. The aim of this study is to determine the accuracy of frozen section in diagnosing BOTs.

Material and methods: This is a retrospective study. A computerized database was used to identify all pathology reports (frozen section analysis and/or definitive histology) with a diagnosis of BOTs performed at our institution between January 2004 and December 2015. Frozen section analysis and definitive histology reports were compared.

Results: 98 BOTs were diagnosed by frozen and/or permanent section pathology in 88 women. The sensitivity of frozen section for diagnosing BOTs was $76.5 \%$; the positive predictive value was $83.3 \%$. The sensitivity of frozen section for diagnosing serous BOTs was significantly higher than for diagnosing mucinous BOTs. Underdiagnosis occurred in $32.7 \%$ of the tumors; it was less frequent in serous tumors than in mucinous tumors. BOTs underdiagnosed at frozen section were significantly larger than those accurately diagnosed at frozen section. In 13 women, frozen section diagnosed BOTs were upgraded to invasive ovarian carcinoma by permanent pathology; there were two relapses in these patients after a mean follow-up of 47.8 months. No recurrence was observed after a mean follow-up of 60.4 months in 19 women with BOTs underdiagnosed as benign tumors at frozen section.
\end{abstract}

Discussion: the surgical management of BOTs based only on intraoperative frozen section diagnosis may result in undertreatment or overtreatment of a substantial number of patients. Under-diagnosis by frozen section is associated with conservative surgery but it does not compromise the outcome in patients with BOTs.

\section{Introduction}

Borderline ovarian tumors (BOTs) are a distinct diagnostic category of epithelial ovarian tumor, distinguished from both benign epithelial ovarian tumors and invasive epithelial ovarian cancers [1]. BOTs account for $5 \%$ of all epithelial ovarian tumors and $15 \%$ of all epithelial ovarian cancers [2]. BOTs are characterized pathologically by features of malignant tumors, including cellular proliferation, stratification of the epithelial lining of the papillae, nuclear atypia, and mitotic activity, but without destructive stromal invasion [3]. They are characterized by earlier stage and younger age at diagnosis, more indolent behavior, longer survival, and later recurrence than seen in cases of invasive ovarian cancer [4].

The preoperative discrimination between the benign cysts, BOTs and invasive cancers is based on patients' characteristics (age, menopausal status), serum markers (mainly CA-125 levels), ultrasonographic and radiological imaging (such as magnetic resonance imaging and positron emission tomography). However, the levels of CA-125 may overlap between patients with benign cysts, BOTs and invasive cancers [5]. Similarly, the imaging findings are not specific of BOTs [6]. Therefore, the diagnosis of BOTs cannot be surely established prior to surgery and intraoperative decisions regarding the surgical management should be based on frozen section analysis.
The accuracy of frozen section analysis is of considerable importance in BOTs. Overdiagnosis of BOTs may results in overtreatment while, in case of BOTs, a conservative, fertility sparing surgery can be carried out in women of reproductive age who wish to retain their fertility. Similarly, it is important to discriminate BOTs from benign cysts; in fact, patients with a BOT misinterpreted as benign tumor may receive an inadequate treatment and staging that result in subsequent additional surgical intervention and possible tumor spread.

Frozen section analysis of ovarian tumors has sensitivity between $65 \%$ and $97 \%$ and specificity between $97 \%$ and $100 \%$ in differentiating invasive and non-invasive specimens [7]. Frozen section analysis of BOTs, however, is notoriously difficult with a significantly lower sensitivity and specificity compared to benign tumors of the ovary and ovarian cancers [8]. Since BOTs comprise only $10 \%$ of epithelial

Correspondence to: Simone Ferrero MD, $\mathrm{PhD}$, Academic Unit of Obstetrics and Gynecology, IRCCS AOU San Martino - IST, Largo R. Benzi 10, 16132 Genoa, Italy, Tel: +01139010 511525; Fax: +01139 010511525; E-mail: simone.ferrero@ unige.it

Key words: accuracy, borderline tumors of the ovary, frozen section, diagnosis, ovarian tumors

Received: March 17, 2018; Accepted: March 29, 2018; Published: April 05, 2018 
ovarian malignancies, studies investigating the accuracy of frozen section analysis of BTOs are characterized by a limited number of patients.

This retrospective study aims to provide additional data on the accuracy of frozen section in diagnosing BOTs.

\section{Materials and methods}

\section{Study design}

In this retrospective study, a computerized database was used to search all pathology reports (frozen section analysis and/or definitive histology) with a diagnosis of BOTs performed at our institution between January 2004 and December 2015. The computerized database contains all the pathology reports performed at our institution. Only cases in which frozen section analysis was performed were considered for the analysis. The frozen section diagnosis was compared with the final paraffin section diagnosis. Institutional review board approval for medical record and pathology report reviews was obtained.

\section{Pathological analysis}

The fresh pathologic materials were sent to the pathology department immediately after excision. All frozen section specimens were analyzed by a pathology resident and a board-certified pathologist, specialized in gynecologic pathology. After the measurement of tumor size, the most suspected areas of adnexal mass and the solid and papillary areas of the tumor wall were chosen for frozen section. Each specimen had at least two representative sections sampled for frozen section analysis. Two to four of the most representative samples were frozen in a cryostat and $5-\mu \mathrm{m}$ sections were taken and stained with hematoxylin and eosin. The number of sections was determined according to the tumor size and the number of solid and suspicious areas of the mass. In all cases, at least two samples were obtained every $1 \mathrm{~cm}$ of maximal tumor diameter for paraffin section examination. Histopathologic diagnosis was performed according to the criteria of World Health Organization; tumors were staged according to the FIGO classification. BOTs were defined as follows: epithelial hyperplasia in the form of stratification, tufting, cribriform and micropapillary arrangements, atypia (usually mild to moderate), detached cell clusters, variable and usually minimal mitotic activity, and absence of destructive stromal invasion [1].

Mucinous tumors were diagnosed using the criteria defined by Hart and Norris [9]. The diagnosis of other tumors was made according to the guidelines of Scully [10].

\section{Surgical procedures}

Data on patients' characteristics and surgical procedures were retrospectively collected. During the study period, fertilitysparing surgery was performed in premenopausal women who desired childbearing; all the other women were treated with total abdominal hysterectomy and bilateral salpingo-oophorectomy, abdominal washing, infracolic omentectomy, peritoneal biopsies and appendectomy in case of mucinous histotype. Systematic lymph node dissection was not performed otherwise in case of intraoperative frozen section diagnosis of invasive tumor.

\section{Statistical analysis}

The Kruskal-Wallis one-way analysis of variance (with Dunn's multiple comparison post hoc test) was used to compare the diameter of tumors with different histology. The Mann-Whitney test was used to compare the diameter of tumors correctly diagnosed or underdiagnosed by frozen section. The $\chi^{2}$ test and the Fisher's exact test were used to compare proportions. Data were archived using Excel 2007 (Microsoft, Redmond, WA, USA) and analyzed using Sigma Stat software version 3.5 (SPSS Science, Chicago, IL, USA). P $<0.05$ was considered statistically significant.

\section{Results}

During the study period, 98 BOTs were diagnosed by frozen and/or permanent section pathology in 88 women.

\section{Characteristics of the study population}

The mean $( \pm$ SD) age of the patients was $47.9 \pm 18.2$ (range 1886) years. Forty-eight women were premenopausal. The mean serum concentration of CA-125 was $53.6 \mathrm{IU} / \mathrm{L}$ (range $3.0-369.0 \mathrm{IU} / \mathrm{L} ; \mathrm{n}=$ 62), of CA-19.9 was $40.8 \mathrm{IU} / \mathrm{L}$ (range $6.2-324.0 \mathrm{IU} / \mathrm{L} ; \mathrm{n}=62$ ) and of CEA was $4.4 \mathrm{ng} / \mathrm{ml}$ (range $0.9-15.0 \mathrm{ng} / \mathrm{ml} ; \mathrm{n}=55$ ). Fifty-eight tumors (59.2\%) were histologically serous, 35 tumors (35.7\%) were mucinous and 5 tumors $(5.1 \%)$ were mixed. The mean $( \pm$ SD) tumor diameter was $11.9 \pm 7.3$ (range, $1.5-32.0) \mathrm{cm}$. The mean $( \pm \mathrm{SD}$ ) diameter of mucinous tumors $(17.1 \pm 7.1$; range, $7.0-32.0 \mathrm{~cm})$ was significantly greater than the diameter of serous tumors $(9.1 \pm 5.8 \mathrm{~cm}$; range, $1.5-$ $24.0 \mathrm{~cm} ; \mathrm{p}<0.05)$ and the diameter of tumors with mixed histology $(7.4$ $\pm 3.4 \mathrm{~cm}$; range $4.0-12.0 \mathrm{~cm} ; \mathrm{p}<0.05)$.

After surgery, 56 patients (63.6\%) were FIGO IA stage, 10 patients (11.3\%) IB, 16 patients (18.2\%) IC ( 3 of them as a consequence of cyst rupture), 2 patients (2.7\%) IIA, 2 patients (2.7\%) IIB, 1 patient $(1.3 \%)$ IIIA, and 1 patient (1.3\%) IIIC. 27 patients had fertility-sparing surgery; the remaining patients underwent a classic stadiation.

Five women had non-invasive tumor implants $(5 / 88 ; 5.7 \%)$ and four women had invasive implants $(4 / 88 ; 4.5 \%)$. Tumor implants were localized in the omentum $(\mathrm{n}=3)$, in the pelvic peritoneum $(\mathrm{n}=$ $4)$, in the pouch of Douglas $(n=1)$ and in the fallopian tube $(n=1)$. Concurrent endometriosis was diagnosed in 7 cases (8.0\%).

\section{Pathological analysis}

Out of 98 tumors included in the study, 78 (79.6\%) were diagnosed to be BOTs at frozen section. The frozen section diagnosis of BOT agreed with permanent pathology diagnosis in 65 of 98 tumors $(66.3 \%$; $95 \%$ C.I. 56.1\%-75.6\%). Using results from paraffin section as a gold standard, the sensitivity of frozen section for diagnosing BOTs was $76.5 \%$ (65/85; $95 \%$ C.I. $66.0 \%$ - 85.0\%); the specificity could not be calculated because this study included only BOTs diagnosed by frozen section and/or permanent pathology. Out of 78 tumors diagnosed as BOTs at frozen section, 65 were confirmed to be BOTs at permanent pathology (positive predictive value, PPV, 83.3\%; 95\% C.I., 73.2\%$90.8 \%)$. The sensitivity of frozen section for diagnosing serous BOTs (86.8\%; 46/53; 95\% C.I., 74.7\%-94.5\%) was significantly higher than the sensitivity of frozen section for diagnosing mucinous BOTs $(59.3 \%$; 16/27; 95\% C.I. $38.8 \%-77.6 \%$; $\mathrm{p}=0.012$ ).

Underdiagnosis occurred in $32.7 \%$ of the tumors (32/98). 19 tumors were interpreted as benign by frozen section and were upgraded to BOTs by permanent pathology. 13 frozen sections diagnosed BOTs were upgraded to invasive ovarian carcinoma by permanent pathology (Table 1). BOTs underdiagnosed by frozen section were significantly larger (mean diameter, $14.1 \pm 7.7 \mathrm{~cm}$ ) than those accurately diagnosed by frozen section $(10.8 \pm 6.9 ; \mathrm{p}=0.035)$. Underdiagnosis by frozen section occurred less frequently in serous tumors $(20.7 \%, 12 / 58)$ than in mucinous tumors $(51.4 \%, 18 / 35 ; \mathrm{p}=0.004)$. 
Overdiagnosis occurred in 1 tumor (1.0\%). One permanent pathology proven BOT was diagnosed as malignant at frozen section. In no case a tumor diagnosed to be BOT at frozen section was found to be benign at permanent pathology (Table 1).

The characteristics of tumors with correct diagnosis at frozen section were compared with those of tumors misdiagnosed at frozen section (Table 2). This analysis confirmed that serous tumors were more frequently correctly diagnosed at frozen section than other histotypes $(p=0.004)$. There was a trend for tumors with larger diameter $\leq 5 \mathrm{~cm}$ to be more frequently correctly diagnosed than those with larger diameter $>5 \mathrm{~cm}$, but the difference did not reach statistical significance $(p=0.086)$. Age of the patients, presence of unilateral of bilateral tumors, spread of the tumors outside the ovary, elevation of tumor markers, study period and presence of endometriosis did not differ between tumors with correct diagnosis at frozen section with those misdiagnosed at frozen section (Table 2).

\section{Management of tumors included in the study}

19 patients had frozen section diagnosis of benign tumor and definitive diagnosis of BTO; none of these tumors was staged during the initial surgery. The mean age of these patients was 43.9 years (range 24-83 years). The histological type of these BOTs was mucinous in 10 patients, serous in 7 patients and mixed in 2 patients. Of these 19 women, 4 underwent second surgery for staging purposes, 15 opted for a conservative approach with a clinical and radiological follow-up.

13 frozen sections diagnosed BOTs were upgraded to invasive ovarian carcinoma by permanent pathology; the clinical outcomes of these patients are shown in (Table 3). Among these 13 patients, seven were premenopausal but only three patients wanted to preserve their fertility. In one of these premenopausal patients the staging for ovarian cancer was actuated for the positive frozen section for mucinous ovarian cancer in the omentum. Only two patients underwent fertility-

Table 1. Frozen diagnosis compared with permanent pathology diagnosis.

\begin{tabular}{|c|c|c|c|c|}
\hline & \multicolumn{3}{|c|}{ Permanent pathology } \\
\hline Frozen section & Benign & Borderline & Malignant \\
\hline Benign & 0 & 19 & 0 \\
\hline Borderline & 0 & 65 & 13 \\
\hline Malignant & 0 & 1 & 0 \\
\hline Total & 0 & 85 & 19 \\
\hline
\end{tabular}

Table 2. Tumor characteristics and accuracy of frozen section diagnosis.

\begin{tabular}{|c|c|c|c|c|c|c|}
\hline & \multicolumn{3}{|c|}{ Frozen diagnosis compared with permanent diagnosis } & \multicolumn{3}{|c|}{ Details of misdiagnosis } \\
\hline & Correct & Misdiagnosis & p value & $\begin{array}{l}\text { Underdiagnosis } \\
\text { BTO-Malignant }\end{array}$ & $\begin{array}{c}\text { Underdiagnosis } \\
\text { Benign-BTO }\end{array}$ & Overdiagnosis \\
\hline Age & & & 0.517 & & & \\
\hline$<35(\mathrm{n}=30)$ & $18(60.0 \%)$ & $12(40.0 \%)$ & & $4(13.3 \%)$ & $8(26.7 \%)$ & $0(0.0 \%)$ \\
\hline$>35(\mathrm{n}=68)$ & $47(69.1 \%)$ & $21(30.9 \%)$ & & $9(13.2 \%)$ & $11(16.2 \%)$ & $1(1.5 \%)$ \\
\hline \multicolumn{7}{|l|}{ Tumor size } \\
\hline & & & 0.086 & & & \\
\hline$>5 \mathrm{~cm}(\mathrm{n}=78)$ & $48(61.5 \%)$ & $30(38.5 \%)$ & & $11(14.1 \%)$ & $18(23.1 \%)$ & $1(1.3 \%)$ \\
\hline \multirow{2}{*}{$\leq 5 \mathrm{~cm}(\mathrm{n}=20)$} & $17(85.0 \%)$ & $3(15.0 \%)$ & & $2(10.0 \%)$ & $1(5.0 \%)$ & $0(0.0 \%)$ \\
\hline & & & 0.200 & & & \\
\hline$>10 \mathrm{~cm}(\mathrm{n}=49)$ & $29(59.2 \%)$ & $20(40.8 \%)$ & & $10(20.4 \%)$ & $10(20.4 \%)$ & $0(0.0 \%)$ \\
\hline \multirow{2}{*}{$\leq 10 \mathrm{~cm}(\mathrm{n}=49)$} & $36(73.5 \%)$ & $13(26.5 \%)$ & & $3(6.1 \%)$ & $9(18.4 \%)$ & $1(2.0 \%)$ \\
\hline & & & 0.501 & & & \\
\hline$>15 \mathrm{~cm}(\mathrm{n}=27)$ & $16(59.3 \%)$ & $11(40.7 \%)$ & & $8(29.6 \%)$ & $3(11.1 \%)$ & $0(0.0 \%)$ \\
\hline$\leq 15 \mathrm{~cm}(\mathrm{n}=71)$ & $49(69.0 \%)$ & $22(31.0 \%)$ & & $5(7.1 \%)$ & $16(22.5 \%)$ & $1(1.4 \%)$ \\
\hline Tumors side & & & 0.199 & & & \\
\hline unilateral $(\mathrm{n}=74)$ & $46(62.2 \%)$ & $28(37.8 \%)$ & & $10(13.5 \%)$ & $17(23.0 \%)$ & $1(1.4 \%)$ \\
\hline bilateral $(n=24)$ & $19(79.2 \%)$ & $5(20.8 \%)$ & & $3(12.5 \%)$ & $2(8.3 \%)$ & $0(0.0 \%)$ \\
\hline Histology & & & 0.004 & & & \\
\hline serous $(\mathrm{n}=58)$ & $46(79.3 \%)$ & $12(20.7 \%)$ & & $5(8.6 \%)$ & $7(12.1 \%)$ & $0(0.0 \%)$ \\
\hline mucinous $(\mathrm{n}=35)$ & $16(45.7 \%)$ & $19(54.3 \%)$ & & $8(22.9 \%)$ & $10(28.6)$ & $1(2.9 \%)$ \\
\hline mixed $(n=5)$ & $3(60.0 \%)$ & $2(40.0 \%)$ & & $0(0.0 \%)$ & $2(40.0 \%)$ & $0(0.0 \%)$ \\
\hline Spread outside ovary & & & 0.901 & & & \\
\hline present $(\mathrm{n}=29)$ & $20(69.0 \%)$ & $9(31.0 \%)$ & & $5(17.2 \%)$ & $4(13.8 \%)$ & $0(0.0 \%)$ \\
\hline absent $(\mathrm{n}=69)$ & $45(65.2 \%)$ & $24(34.8 \%)$ & & $8(11.6 \%)$ & $15(21.7 \%)$ & $1(1.4 \%)$ \\
\hline Tumor markers & & & 0.840 & & & \\
\hline elevation $*(n=35)$ & $21(60.0 \%)$ & $14(40.0 \%)$ & & $9(25.7 \%)$ & $5(14.3 \%)$ & $0(0.0 \%)$ \\
\hline normal $(\mathrm{n}=27)$ & $16(59.3 \%)$ & $11(40.7 \%)$ & & $3(11.1 \%)$ & $8(29.6 \%)$ & $0(0.0 \%)$ \\
\hline Study period & & & 0.889 & & & \\
\hline $2004-2009(\mathrm{n}=51)$ & $34(66.7 \%)$ & $17(33.3 \%)$ & & $8(15.7 \%)$ & $9(17.6 \%)$ & $0(0.0 \%)$ \\
\hline $2010-2015(n=47)$ & $31(66.0 \%)$ & $16(34.0 \%)$ & & $5(10.6 \%)$ & $10(21.3 \%)$ & $1(2.1 \%)$ \\
\hline Endometriosis & & & 0.714 & & & \\
\hline present $(n=8)$ & $6(75.0 \%)$ & $2(25.0 \%)$ & & $1(12.5 \%)$ & $1(12.5 \%)$ & $0(0.0 \%)$ \\
\hline absent $(n=90)$ & $59(65.6 \%)$ & $31(34.4 \%)$ & & $12(13.3 \%)$ & $18(20.0 \%)$ & $1(1.1 \%)$ \\
\hline
\end{tabular}

* defined as CA125>35U/ml; CA $19.9>37 \mathrm{U} / \mathrm{ml}$ 
Table 3. Patient's characteristics and outcomes of Underdiagnosis.

\begin{tabular}{|c|c|c|c|c|c|c|c|c|c|}
\hline $\begin{array}{c}\text { BOT } \\
\text {-MALIGNANT }\end{array}$ & Age & \begin{tabular}{|c|} 
Tumor diameter \\
$(\mathbf{c m})$
\end{tabular} & First surgery & FIGO stage & Re-staging & $\begin{array}{l}\text { Adjuvant } \\
\text { therapy }\end{array}$ & Recurrence & Follow-up & Notes \\
\hline \multicolumn{10}{|l|}{$\begin{array}{l}\text { Serous } \\
(\mathrm{n}=5)\end{array}$} \\
\hline P.C & 75 & 5,5 & Complete & Ia & No & No & No & 46 months & $\begin{array}{l}\text { Death for other } \\
\text { causes }\end{array}$ \\
\hline U.A & 31 & 2 & Complete, LN & IIIc & No & Yes & Yes 33 months & 33 months & $\begin{array}{l}\text { Frozen section } \\
\text { in omentum } \\
\text { positive. }\end{array}$ \\
\hline B.M & 61 & 4 & Complete & Ia & No & No & No & 40 months & \\
\hline P.E & 41 & 4 & Complete & Ic & Yes & Yes & No & 62 months & $\begin{array}{l}\text { Rising tumor } \\
\text { markers }\end{array}$ \\
\hline R.M & 39 & 14 & Complete & Ic & Yes & Yes & No & 31 months & \\
\hline \multicolumn{10}{|l|}{$\begin{array}{l}\text { Mucinous } \\
(\mathbf{n}=\mathbf{8})\end{array}$} \\
\hline C.L & 37 & 21 & Complete, LN & Ia & No & No & No & 60 months & \\
\hline P.G & 49 & 22 & Complete, LN & IIa & Yes & Yes & Yes 55 months & 57 months & \\
\hline M.M & 58 & 36 & Complete & $\mathrm{Ib}$ & No & Yes & No & 70 months & \\
\hline S.A & 26 & 18 & Fertility sparing & Ia & No & No & No & 84 months & \\
\hline P.O & 67 & 29 & Complete & Ia & No & No & No & 59 months & $\begin{array}{l}\text { Death for other } \\
\text { causes }\end{array}$ \\
\hline C.C & 33 & 23 & Fertility sparing & Ia & No & Yes & No & 42 months & \\
\hline D.O & 46 & 17 & Complete & Ia & No & No & No & 32 months & \\
\hline M.F & 48 & 28 & Complete & $\mathrm{Ib}$ & Yes & Yes & No & 6 months & \\
\hline
\end{tabular}

preserving surgery. Four patients underwent a restaging procedure and seven patients received adjuvant chemotherapy. There were two relapses; in addition, one patient has rising CA 19.9 and CA125 (40 I.U./l and 45 I.U./l respectively), but at the moment she has no clinical relapse. The mean follow-up is 47.8 months (31-84 months).

In the fifteen patients with BOTs underdiagnosed as benign tumors at frozen section that did not undergo restaging surgery, the mean follow-up was 60.4 months (38 - 100 months). No recurrence was observed.

\section{Discussions}

The appropriate surgical management of ovarian tumors requires accurate histological diagnosis. Benign lesions are usually managed conservatively, and a similar approach may be used in some patients with BOTs who wish to preserve fertility. Although advanced-stage ovarian cancer is usually evident at the time of surgery, the distinction of benign, borderline, and malignant tumors macroscopically confined to the ovaries is more difficult [11]. Frozen section diagnosis offers an important and helpful adjunct to the intra-operative diagnosis and has greatly impacted the care of gynecologic oncology patients. It also helps to determine the staging, as well as the appropriate surgical technique for each case [12]. Intra-operative diagnosis is especially important in women of reproductive age, in which fertility preservation is of paramount importance.

The accuracy of frozen section examination in diagnosing ovarian tumors is greater than $90 \%$ [7]. However, the accuracy of frozen section is lower in diagnosing BOTs [13]. In a review of the literature including only studies assessing the accuracy of intraoperative frozen section analysis in diagnosing BOTs (317 women), Tempfer et al. found that intraoperative frozen section has a sensitivity of $71.1 \%$ and a PPV of $84.3 \%$ [14]. Overdiagnosis and underdiagnosis occurred in $6.6 \%$ and $30.6 \%$ of the cases, respectively. We performed a literature research and identified five previous studies exclusively examining the frozen section diagnosis of BOTs (12-16). Data of these studies and the present investigation were pooled; 516 cases were identified. Table 4 shows that agreement between frozen section diagnosis and definitive
Table 4. Frozen section diagnosis of BOTs compared with permanent histology, individual data of six studies and pooled analysis.

\begin{tabular}{|c|c|c|c|}
\hline \multirow[b]{2}{*}{ Frozen section } & \multicolumn{3}{|c|}{ Permanent pathology } \\
\hline & Benign & Borderline & Malignant \\
\hline \multicolumn{4}{|c|}{ Menzin et al. (1995) } \\
\hline Benign & 0 & 9 & 0 \\
\hline Borderline & 3 & 23 & 13 \\
\hline Malignant & 0 & 0 & 0 \\
\hline \multicolumn{4}{|c|}{ Houck et al. (2000) } \\
\hline Benign & 0 & 35 & 0 \\
\hline Borderline & 4 & 84 & 6 \\
\hline Malignant & 0 & 11 & 0 \\
\hline \multicolumn{4}{|c|}{$\begin{array}{l}\text { Kayikcioglu et al. } \\
\text { (2000) }\end{array}$} \\
\hline Benign & 0 & 3 & 0 \\
\hline Borderline & 3 & 23 & 4 \\
\hline Malignant & 0 & 0 & 0 \\
\hline \multicolumn{4}{|l|}{$\begin{array}{l}\text { Tempfer et al. } \\
\text { (2007) }\end{array}$} \\
\hline Benign & 0 & 23 & 0 \\
\hline Borderline & 0 & 69 & 4 \\
\hline Malignant & 0 & 0 & 0 \\
\hline \multicolumn{4}{|c|}{ Kim et al. (2009) } \\
\hline Benign & 0 & 24 & 0 \\
\hline Borderline & 5 & 63 & 8 \\
\hline Malignant & 0 & 1 & 0 \\
\hline \multicolumn{4}{|l|}{ Current study } \\
\hline Benign & 0 & 19 & 0 \\
\hline Borderline & 0 & 65 & 13 \\
\hline Malignant & 0 & 1 & 0 \\
\hline \multicolumn{4}{|l|}{ Pooled analysis } \\
\hline Benign & 0 & 113 & 0 \\
\hline Borderline & 15 & 327 & 48 \\
\hline Malignant & 0 & 13 & 0 \\
\hline
\end{tabular}

histology was observed in 327/516 (63.4\%) patients, yielding an overall sensitivity of $72.2 \%$ and a PPV of $83.3 \%$ (Figure 1). Overdiagnosis and underdiagnosis were identified in 28/516 (5.4\%) and in 161/516 $(31.2 \%)$ cases, respectively. 
The results of our study demonstrate that the low sensitivity of frozen section examination is mainly due to the underdiagnosis of mucinous BOTs as benign tumors (Figures 1 and 2). The inaccuracy of frozen section examinations in diagnosing mucinous BOTs was previously reported by other authors [13-16]. The limitations of frozen section in diagnosing mucinous BOTs may be due to the fact that, in this neoplasm, invasive mucinous ovarian cancer can often be observed in areas adjacent to benign and borderline histology, suggesting a continuum to malignant progression which is not observed in the other epithelial ovarian lesions [17]. Adequate sampling in frozen section cannot sometimes be performed in mucinous tumors due to relatively larger size; therefore, malignant areas can be overlooked. Several studies showed that mucinous histology, tumor size and experience of the pathologist influences the accuracy of frozen section diagnosis $[18,19]$. Performing multiple sections in large tumors may increase the possibility to identify focal borderline changes. Tempfer et al. [14] observed that three out of four misread tumors have a diameter $\geq 5 \mathrm{~cm}$; therefore, they suggested that a cut-off of $5 \mathrm{~cm}$ for tumor diameter is reasonable for recommending multiple sections. In our series, BOTs underdiagnosed at frozen section were significantly larger than those accurately diagnosed at frozen section. In addition, there was a trend for tumors with diameter $\leq 5 \mathrm{~cm}$ to be less frequently misdiagnosed than tumors with diameter $>5 \mathrm{~cm}$, but the difference did not reach statistical significance (Table 2). In agreement with these observations,
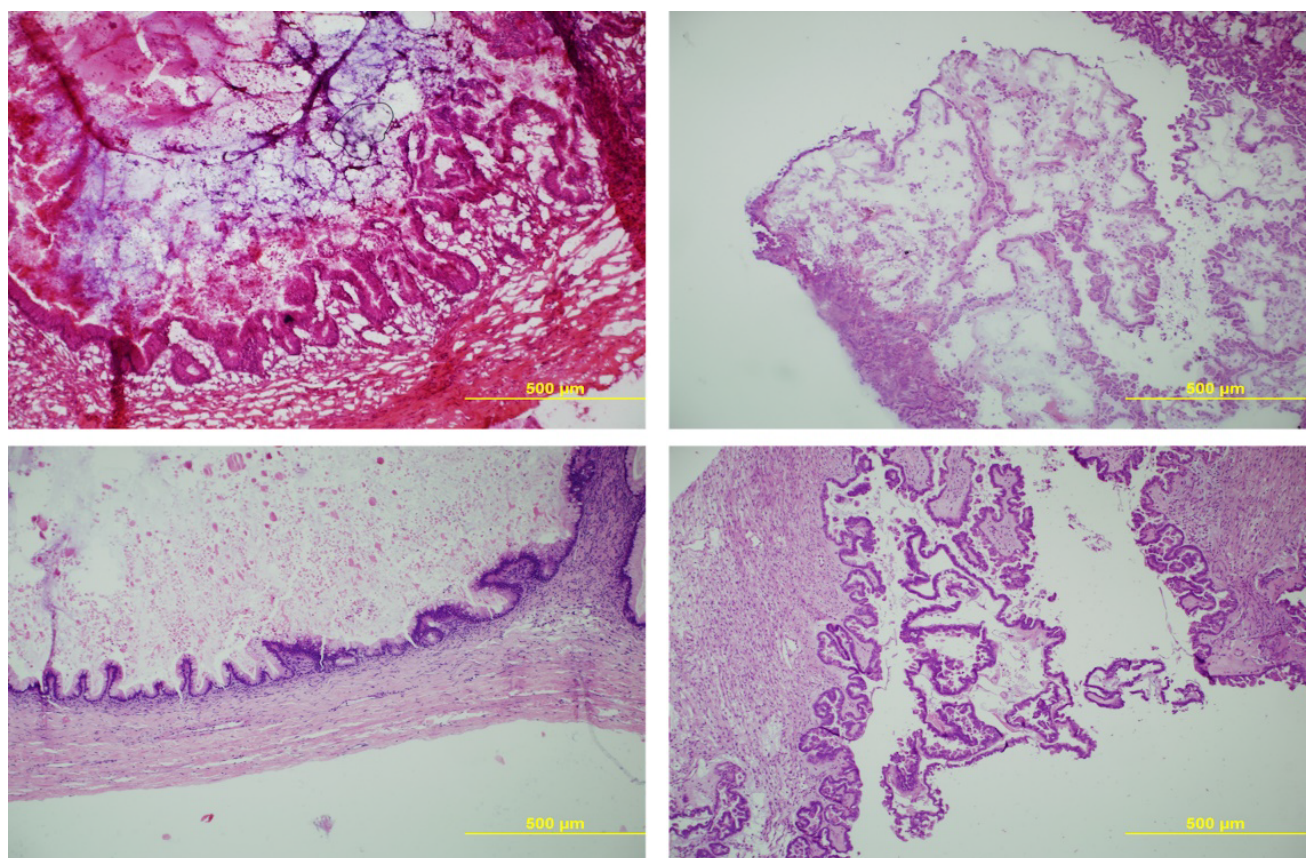

Figure 1. (100x) Upper Left: Frozen Section examination of a mucinous borderline tumor of intestinal type; Lower Left: Formalin Fixed - Paraffin Embedded Definitive slide of the same sample of a mucinous borderline tumor of intestinal type; Upper Right: Frozen Section examination of a serous borderline tumor; Lower Right: Formalin Fixed - Paraffin Embedded Definitive slide of the same sample of a serous borderline tumor.


Figure 2. (200x) Upper Left: Frozen Section examination of a mucinous borderline tumor of intestinal type; Lower Left: Formalin Fixed - Paraffin Embedded Definitive slide of the same sample of a mucinous borderline tumor of intestinal type; Upper Right: Frozen Section examination of a serous borderline tumor. 
misdiagnosis occurred in $15.0 \%$ of tumors with diameter $\leq 5 \mathrm{~cm}$, in $26.5 \%$ of tumors with diameter $\leq 10 \mathrm{~cm}$ and in $31.0 \%$ of tumors with diameter $\leq 15 \mathrm{~cm}$.

In our series, the majority of the diagnostic discrepancies were caused by underdiagnosis and the most common single error, accounting for $43.8 \%$ of the cases (14/32), was the misinterpretation of BOTs as benign mucinous cystadenoma. Such cases present a potential clinical dilemma because these patients may not undergo the formal staging (omental biopsy or omentectomy, with or without peritoneal biopsy) that would normally follow a correct intraoperative diagnosis of BOT. In our practice, the management options (surgical restaging or follow-up) of these patients were determined on individual basis depending on the overall clinical, pathological, and operative findings.

In keeping with previous reports $[8,20]$, we found that the intraoperative assessment of serous BOTs was more reliable than in mucinous BOTs with $86.8 \%$ of the cases accurately diagnosed.

Although patients with BOTs have an excellent prognosis, there is a risk of recurrence [21]. The overall false-positive rate in our series was $1.0 \%$ similar to the range $(0.0 \%-2.2 \%)$ reported in previous studies $[8,22,23]$. Patients with ovarian carcinoma (but not those with BOTs) in our institute usually undergo unilateral retroperitoneal lymphadenectomy; consequently, this type of intraoperative error may lead to extended and unnecessary surgery.

Therefore, the reliability of frozen-section diagnosis of BOTs is a critical determinant in the selection of the appropriate surgical procedure [24].

In the current study, the clinical outcome after misdiagnosis at frozen section was reported. No recurrence was observed in the 19 patients with BOTs that were interpreted as benign at frozen section. Retrospective studies demonstrated that there is no difference in recurrence rates between women who undergo restaging of BOTs and those who do not [25-28]. Restaging procedure does not seem to have a significant impact on the management of patients diagnosed with BOTs, especially in mucinous subtype [28]. Our data are in agreement with those published by Kim et al. [29] and suggest that under diagnosis by frozen section examination does not compromise the outcome in patients with BOTs. In fact, although underdiagnosis was associated with conservative surgery, it did not cause treatment failure. These findings suggest that the extent of surgery is not the critical factor for outcome in women with BOTs.

In conclusion, the intraoperative management of BOTs needs a histological and surgical evaluation. An intraoperative assessment must be combined with a direct communication between surgeon and pathologist and both physicians should know the limitations of this examination. In particular, surgeons should be aware that frozen section diagnosis is based on the assessment of two to three sections from the most suspicious portion of the tumour, while the final diagnosis is made after the evaluation of an internationally agreed standard of a minimum of one section per $\mathrm{cm}$ of maximal tumour diameter [30]. The surgical management of BOTs based only on intraoperative frozen section diagnosis should be used with caution because this strategy may result in undertreatment of a substantial number of patients or overtreatment in few cases. However, when frozen section examination diagnose BOT in patients who do not with to preserve fertility, the surgeon should perform a standard staging [31]. In these cases, large peritoneal biopsies and deperitonealisation of all suspicious regions are required [32]. Obviously, a close follow-up is required in women who undergo a conservative treatment [33].

\section{Authorship}

S.F. conceived and designed the study, performed the interpretation of data, and drafted and revised the manuscript. F.B. acquired and analyzed the data and drafted the manuscript. V.G.V. performed the histopatological examination V.G.V, P.L.V. and L.P. revised the manuscript. All authors approved the final version of the manuscript.

\section{Funding}

No funding was received to prepare this review.

\section{Conflict of interest}

The authors have no financial, personal or competing interests.

\section{References}

1. Serov SF, Scully RE, Sobin LH (1973) The World Health Organization International Histological Classification of Ovarian Tumors: histological typing of ovarian tumors. Geneva, Switzerland: World Health Organization, 37-38.

2. Scully RE (1975) World Health Organization classification and nomenclature of ovarian cancer. Natl Cancer Inst Monogr 42: 5-7. [Crossref]

3. Acs G (2005) Serous and mucinous borderline (low malignant potential) tumors of the ovary. Am J Clin Pathol 123: S13-57. [Crossref]

4. Tinelli R, Tinelli A, Tinelli FG, Cicinelli E, Malvasi A (2006) Conservative surgery for borderline ovarian tumors: a review. Gynecol Oncol 100: 185-91. [Crossref]

5. Eltabbakh GH, Yadav PR, Morgan A (1999) Clinical picture of women with early stage ovarian cancer. Gynecol Oncol 75: 476-479. [Crossref]

6. Lalwani N, Shanbhogue AK, Vikram R, Nagar A, Jagirdar J, et al. (2010) Current update on borderline ovarian neoplasms. AJR Am J Roentgenol 194: 330-336. [Crossref]

7. Geomini P, Bremer G, Kruitwagen R, Mol BW (2005) Diagnostic accuracy of frozen section diagnosis of the adnexal mass: a metaanalysis. Gynecol Oncol 96: 1-9. [Crossref]

8. Rose PG, Rubin RB, Nelson BE, Hunter RE, Reale FR (1994) Accuracy of frozensection (intraoperative consultation) diagnosis of ovarian tumors. Am J Obstet Gynecol 171: 823-826. [Crossref]

9. Hart WR, Norris HJ (1973) Borderline and malignant mucinous tumors of the ovary. Histologic criteria and clinical behavior. Cancer 31: 1031-1045. [Crossref]

10. Scully RE (1979) Atlas of tumor pathology. Tumors of the ovary and maldeveloped gonads. Washington, DC: Armed Forces Institute of Pathology.

11. Stewart CJ, Brennan BA, Hammond IG, Leung YC, McCartney AJ (2006) Intraoperative assessment of ovarian tumors: a 5-year review with assessment of discrepant diagnostic cases. Int J Gynecol Pathol 25: 216-222. [Crossref]

12. Kayikcioglu F, Pato O, Cengiz S, Tulunay G, Boran N, et al. (2000) Accuracy of frozen section diagnosis in borderline ovarian malignancy. Gynecol Obstet Invest 49: 187-189. [Crossref]

13. Houck K, Nikrui N, Duska L, Chang Y, Fuller AF, et al. (2000) Borderline tumours of the ovary: correlation of frozen and permanent histopathologic diagnosis. Obstet Gynecol 95: 839-843. [Crossref]

14. Tempfer CB, Polterauer S, Bentz EK, Reinthaller A, Hefler LA (2007) Accuracy of intraoperative frozen section analysis in borderline tumors of the ovary: a retrospective analysis of 96 cases and review of the literature. Gynecol Oncol 107: 248-252. [Crossref]

15. Menzin AW, Rubin SC, Noumoff JS, LiVolsi VA (1995) The accuracy of a frozen section diagnosis of borderline ovarian malignancy. Gynecol Oncol 59: 183-185. [Crossref]

16. Kim JH, Kim TJ, Park YG, Lee SH, Lee CW, et al. (2009) Clinical analysis of intraoperative frozen section proven borderline tumors of the ovary. J Gynecol Oncol 20: 176-180. [Crossref]

17. Frumovitz M, Schmeler KM, Malpica A, Sood AK, Gershenson DM (2010) Unmasking the complexities of mucinous ovarian carcinoma. Gynecol Oncol 117: 491- 496. [Crossref]

18. Baker P, Oliva E (2008) A practical approach to intraoperative consultation in gynecological pathology. Int J Gynecol Pathol 27: 353-365. [Crossref] 
19. Brun JL, Cortez A, Rouzier R, Callard P, Bazot M, et al. (2008) Factors influencing the use and accuracy of frozen section diagnosis of epithelial ovarian tumors. Am J Obstet Gynecol 199: 244-247. [Crossref]

20. Wang KG, Chen TC, Wang TY, Yang YC, Su TH (1998) Accuracy of frozen section diagnosis in gynecology. Gynecol Oncol 70:105-110. [Crossref]

21. Ren J, Peng Z, Yang K (2008) A clinicopathologic multivariate analysis affecting recurrence of borderline ovarian tumors. Gynecol Oncol 110: 162-167. [Crossref]

22. Acs G (2002) Intraoperative consultation in gynaecologic pathology. Semin Diagn Pathol 19: 237-254. [Crossref]

23. Puls L, Heidtman E, Hunter JE, Crane M, Stafford J (1997) The accuracy of frozen section by tumor weight for ovarian epithelial neoplasms. Gynecol Oncol 67: 16-19. [Crossref]

24. Wootipoom V, Dechsukhum C, Hanprasertpong J, Lim A (2006) Accuracy of intraoperative frozen section in the diagnosis of ovarian tumors. J Med Assoc Thai 89: 577-582. [Crossref]

25. Camatte S, Morice P, Thoury A, Fourchotte V, Pautier P, et al (2004) Impact of surgical staging in patients with macroscopic "stage I" ovarian borderline tumours: analysis of a continuous series of 101 cases. Eur J Cancer 40: 1842-1849. [Crossref]

26. Fauvet R, Boccara J, Dufournet C, David-Montefiore E, Poncelet C, et al (2004) Restaging surgery for women with borderline ovarian tumors: results of a French multicenter study. Cancer 100: 1145-1151. [Crossref]
27. Winter WE III, Kucera PR, Rodgers W, McBroom JW, Olsen C, et al (2002) Surgical staging in patients with ovarian tumors of low malignant potential. Obstet Gynecol 100: 671-676. [Crossref]

28. Zapardiel I, Rosenberg P, Peiretti M, Zanagnolo V, Sanguineti F et al. (2010) The role of restaging borderline ovarian tumors: Single institution experience and review of the literature. Gynecol Oncol 119: 274-277. [Crossref]

29. Kim K, Chung HH, Kim JW, Park NH, Song YS, et al. (2009) Clinical impact of underdiagnosis by frozen section examination is minimal in borderline ovarian tumors. Eur $J$ Surg Oncol 35: 969-973. [Crossref]

30. The Royal College of Pathologists (2008) Standards and datasets for reporting cancers London: Royal College of Pathologists.

31. Kokka F, Singh N, Reynolds K, Oram D, Jeyarajah A, et al. (2009) The accuracy of frozen section diagnosis in apparent early ovarian cancer results from a UK centre. Histopathology 55: 756-758.

32. Fotopoulou C, Schumacher G, Schefold JC, Denkert C, Lichtenegger W, et al. (2009) Systematic evaluation of the intraoperative tumor pattern in patients with borderline tumor of the ovary. Int J Gynecol Cancer 19: 1550-1555. [Crossref]

33. Lenhard MS, Mitterer S, Kümper C, Stieber P, Mayr D, et al. (2009) Long-term followup after ovarian borderline tumor: relapse and survival in a large patient cohort. Eur $J$ Obstet Gynecol Reprod Biol 145: 189-194. [Crossref]

Copyright: $\odot 2018$ Ferrero S. This is an open-access article distributed under the terms of the Creative Commons Attribution License, which permits unrestricted use, distribution, and reproduction in any medium, provided the original author and source are credited. 